\title{
Mutant mice challenged as models of injury in the central nervous system
}

There is only limited axon regeneration after injury of the brain or the spinal cord, and three major myelin-associated inhibitors-Nogo, myelin-associated glycoprotein (MAG) and oligodendrocyte myelin glycoprotein (OMgp)—have seemed to be partly responsible for this failure. Although in vivo inhibition of Nogo with specific antibodies has shown promising results, the role of Nogo, MAG and OMgp, and a possible synergistic effect between the three, in axon regeneration remains unknown. Jae $\mathrm{K}$. Lee et al. ${ }^{1}$ aim to address this issue with mice lacking the genes encoding the three proteins. Deletion of any single inhibitor resulted in sprouting of healthy and injured axons, but there was no axon regeneration or behavioral improvement, and no synergistic effect in triple mutants-suggesting a limited therapeutic potential of these proteins to treat nervous system injury. Another recent study ${ }^{2}$, however, reports major regeneration and functional repair with a very similar mice model. We asked the experts to discuss these opposing studies.

\section{Martin Schwab:}

Current therapy for spinal cord injuries includes surgical stabilization, anti-inflammatory medication and rehabilitative training. Also, there are two drugs that are currently in clinical trials to treat acute spinal cord injury. Blockage of the intracellular signal transducer rho-a negative intracellular regulator of growth-with the drug Cethrin has shown a neuroprotective role in animal models, and Nogo-A-specific antibodies also seemed promising in a clinical trial. Extensive animal experiments, from rodents to monkeys, in several independent labs have shown that Nogo-specific antibodies, Nogo receptor-blocking peptides and blockade of Nogo signal transduction can enhance regeneration, plasticity and functional recovery.

Using mice triply deficient for Nogo, MAG and OMgp, Cafferty et al. ${ }^{2}$ previously showed major functional repair after partial spinal cord lesions, whereas Lee et al. ${ }^{1}$ found that single, double or triple inactivation of these proteins enhances shortdistance regenerative sprouting after spinal cord injury but does not improve long-distance fiber growth or function. In general, knocking out the genes may result in minor or no physiological effects, whereas acute pharmacological inactivation may induce major changes. This is mainly due to the strong drive for functional compensation, which can additionally be influenced by the mouse genetic background. Previously, Nogo-deficient mice from three labs showed strong, mild or no axon regeneration, probably owing to compensation and strain effects ${ }^{3}$. Controversial results are now shown for the triple knockouts lacking Nogo-A, MAG and OMgp. One caveat is that neither the compensatory upregulation of other inhibitory factors, such as semaphorins, ephrins or proteoglycans, which can restore growth inhibition, nor the role of mouse strain backgrounds were analyzed in the studies.

Conventional gene knockout techniques inactivate a gene from early development throughout its entire life. The lack of major fiber regeneration and behavioral improvement in some Nogo-deficient mice may be another example where well-timed functional suppression of a protein with drugs at the time of central nervous system injury can provide better results for the treatment of a disease than lifelong gene deletions.

Chair, Brain Research Institute, University of Zurich and Swiss Federal Institute of Technology, Zurich, Switzerland.

\section{Conventional gene knockout mice show} severe limitations for the development of therapeutic approaches to spinal cord injury, brain injury or stroke. -Martin Schwab

\section{Mark H. Tuszynski:}

Previous reports have found that therapies that neutralize myelinassociated inhibitors have either dramatic effects on axonal regeneration ${ }^{2}$ or, alternatively, little to no effect ${ }^{4}$. Lee et al. ${ }^{1}$ report now modest effects of inhibiting myelin-associated molecules on axonal growth after injury of the central nervous system.

The authors simultaneously deleted in mice three of the six known myelin associated inhibitors-Nogo, MAG and OMgp but not netrin, semaphorin or ephrin - and found no regeneration of corticospinal axons below the lesion or functional recovery ${ }^{1}$. Only modest sprouting of spared axons was observed, which represents an unfortunate finding, as the corticospinal system is crucial to voluntary motor function in humans. The authors concluded that Nogo, MAG and OMgp do not have a role in axonal regeneration failure, although the interpretation of these findings may be more subtle, for several reasons. First, Nogo, MAG and OMgp could still be involved in regeneration-knocking out their expression but not that of other myelin-associated inhibitors may be insufficient to achieve regeneration of injured axons. Second, knockdown of any molecule during development may alter neural development or generate compensatory changes in ways that are as yet unknown. It remains possible, however, that blocking these same molecules with antibodies in adulthood would have positive effects.

It may also be the case that one has to neutralize more components, other than adult myelin, to achieve regeneration. For example, proteoglycans in the extracellular matrix also potently inhibit axon growth, and combined approaches that target both myelin- and extracellular matrix-associated inhibitors may yield synergistic effects on axon regeneration.

Professor of Neurosciences and Director, Center for Neural Repair, University of California, San Diego, California, USA.

1. Lee, J.K. et al. Assessing spinal axon regeneration and sprouting in Nogo-, MAG- and OMgp-deficient mice. Neuron 66, 663-670 (2010).

2. Cafferty, W.B. et al. MAG and OMgp synergize with Nogo-A to restrict axonal growth and neurological recovery after spinal cord trauma. J. Neurosci. 30, 6825-6837 (2010).

3. Dimou, L. et al. Nogo-A-deficient mice reveal strain-dependent differences in axonal regeneration. J. Neurosci. 26, 5591-5603 (2006).

4. Zheng, B. et al. Lack of enhanced spinal regeneration in Nogo-deficient mice. Neuron 38, 213-224 (2003). 